\title{
Letter to the editor re: "Diagnostic performance of stress myocardial perfusion imaging for coronary artery disease: a systematic review and meta-analysis" - unfair study selection in meta-analysis
}

\author{
Nicola Gaibazzi \\ Received: 16 June 2012 / Revised: 9 July 2012 / Accepted: 12 July 2012 /Published online: 30 August 2012 \\ (C) European Society of Radiology 2012
}

\section{Dear Editor,}

I was disappointed to realise that in the recent meta-analysis published online ahead of print in your journal, entitled "Diagnostic performance of stress myocardial perfusion imaging for coronary artery disease: a systematic review and meta-analysis" by de Jong et al. [1], the single numerically most relevant prospective diagnostic study in the contrast stress-echo field, which I co-authored [2], comprising 400 patients and published in 2010 (in time for being included), was not cited and its data not included in the meta-analysis; this is a relevant mistake, first made by the authors in the data selection process and then by the reviewers in accepting such severely incomplete data: inevitably this makes the meta-analysis results and conclusions deeply flawed.

All inclusion/exclusion criteria, clearly stated in the methods section, are fulfilled by our above-mentioned study, and it could not be otherwise, since that is one of the few prospective and multicentre studies in the contrast stress-echocardiography field, conducted on patients with a previous clinical indication to angiography, so that verifica- tion bias is not an issue. Data regarding stress myocardial perfusion accuracy were easily available in numerical table format and readily at hand in our study. So, can we accept conclusions from a comparison meta-analysis when the quantitatively most relevant contrast-echo study (for sample numerosity second only to one of the CMR studies among all selected studies) has been arbitrarily excluded without an apparent reason? How can we rely on the SPECT and MRI data selection also?

\section{References}

1. de Jong MC, Genders TS, van Geuns RJ, Moelker A, Hunink MG (2012) Diagnostic performance of stress myocardial perfusion imaging for coronary artery disease: a systematic review and metaanalysis. Eur Radiol doi:10.1007/s00330-012-2434-1

2. Gaibazzi N, Rigo F, Reverberi C (2010) Detection of coronary artery disease by combined assessment of wall motion, myocardial perfusion and coronary flow reserve: a multiparametric contrast stressechocardiography study. J Am Soc Echocardiogr 23:1242-1250
N. Gaibazzi $(\bowtie)$

Cardiology Department, Parma University Hospital,

Via Gramsci, 14,

43124 Parma, Italy

e-mail: ngaibazzi@gmail.com 